Canadian

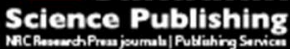

Applied Physiology, Nutrition, and Metabolism Physiologie appliquée, nutrition et métabolisme

\title{
Temporal and bidirectional associations between physical activity and sleep in primary school-aged children
}

\begin{tabular}{|r|l|}
\hline Journal: & Applied Physiology, Nutrition, and Metabolism \\
\hline Manuscript ID & apnm-2016-0424.R2 \\
\hline Manuscript Type: & Article \\
\hline Complete List of Authors: & $\begin{array}{l}\text { Vincent, Grace; Central Queensland University, Appleton Institute } \\
\text { Barnett, Lisa; Deakin University School of Health and Social Development } \\
\text { Lubans, David; University of Newcastle, Priority Research Centre for } \\
\text { Physical Activity and Nutrition } \\
\text { Salmon, Jo; Deakin University, Institute for Physical Activity and Nutrition } \\
\text { (IPAN) } \\
\text { Timperio, Anna; Deakin University, Institute for Physical Activity and } \\
\text { Nutrition (IPAN) } \\
\text { Ridgers, Nicola; Deakin University, Institute for Physical Activity and } \\
\text { Nutrition }\end{array}$ \\
\hline Keyword: & \begin{tabular}{l} 
physical activity < exercise, Accelerometry, Youth, SenseWear \\
\hline
\end{tabular} \\
\hline
\end{tabular}




\section{Temporal and bidirectional associations between physical activity and sleep in primary school-aged children}

Grace E. Vincent ${ }^{1}$, Lisa M. Barnett ${ }^{2}$, David R. Lubans ${ }^{3}$, Jo Salmon ${ }^{4}$, Anna Timperio ${ }^{4}$ and Nicola D. Ridgers ${ }^{4}$

${ }^{1}$ Central Queensland University, Appleton Institute, 44 Greenhill Road, Wayville, SA 5034, Australia; Email: g.vincent@,cqu.edu.au

${ }^{2}$ School of Health and Social Development, Deakin University, 221 Burwood Highway, Burwood, VIC 3125, Australia; Email: lisa.barnett@deakin.edu.au

${ }^{3}$ University of Newcastle, Priority Research Centre for Physical Activity and Nutrition, University Drive, Callaghan NSW 2308, Australia; Email: David.Lubans@,newcastle.edu.au

${ }^{4}$ Deakin University, Geelong, Australia, Institute for Physical Activity and Nutrition (IPAN), School of Exercise and Nutrition Sciences, Australia; Emails: jo.salmon@deakin.edu.au; anna.timperio@deakin.edu.au; nicky.ridgers@deakin.edu.au [Corresponding author]; Tel: +61392446718 [N Ridgers]

Number of words: 3029 words

Figures: 0

Tables: 2 


\begin{abstract}
Purpose: The directionality of the relationship between children's physical activity and sleep is unclear. This study examined the temporal and bidirectional associations between objectively-measured physical activity, energy expenditure and sleep in primary school-aged children.

Methods: A sub-group of children $(\mathrm{n}=65$, aged $8-11$ years $)$ from the Fitness, Activity and Skills Testing (FAST) Study conducted in Melbourne, Australia, had their sleep and physical activity assessed using the SenseWear Pro Armband for eight consecutive days. Outcome measures included time spent in light- (LPA), moderate- to vigorous-intensity physical activity (MVPA), activity energy expenditure (AEE), time in bed, total sleep time and sleep efficiency. Multilevel analyses were conducted using generalized linear latent mixed models to determine whether physical activity on one day was associated with sleep outcomes that night, and whether sleep during one night was associated with physical activity the following day.
\end{abstract}

Results: No significant associations were observed between time in bed, total sleep time, and sleep efficiency with LPA, MVPA and AEE in either direction.

Conclusion: This study found no temporal or bidirectional associations between objectivelymeasured physical activity, AEE and sleep. Future research is needed to understand other sleep dimensions that may impact on or be influenced by physical activity to provide potential intervention targets to improve these outcomes.

Key words: Physical activity; accelerometry; youth; SenseWear 


\section{Introduction}

Current recommendations state children should accumulate 60 minutes of moderate- to vigorous-intensity physical activity (MVPA) per day (World Health Organization 2010) and obtain a total sleep time of 9-11 h per night (National Sleep Foundation 2011). Not meeting both of these recommendations is associated with increased cardio-metabolic risk (Andersen et al. 2006; Spruyt et al. 2011) and overweight/obesity (Sekine et al. 2002; Taheri 2006; Touchette et al. 2008). Over the last century, there have been consistent rapid declines in children's physical activity levels (Dollman et al. 2005) and sleep duration (Matricciani et al. 2012). Given the public health importance of sufficient physical activity, energy expenditure and sleep (Hillman et al. 2013; Janssen and LeBlanc 2010; Levine 2003) a greater understanding of the association and directionality of this relationship is needed for designing interventions to improve both of these behaviours in children. For example, does increasing daily physical activity benefit sleep, and/or does improving sleep duration enhance daily physical activity levels?

A recent experimental study reported that children watched more television and had lower daytime activity counts per epoch when sleep was decreased by $1.5 \mathrm{~h} /$ night for 1 week (compared with increased; Hart et al. 2016). However, perhaps not surprisingly total accelerometer-measured activity counts while awake were higher when sleep was decreased (i.e., children spent more time awake) than when sleep was increased (Hart et al. 2016). However, observational studies that have utilised objective measures to examine temporal and bidirectional relationships between children's physical activity and sleep are equivocal (Ekstedt et al. 2013; Krietsch et al. 2016; Mcneil et al. 2015; Pesonen et al. 2011; Sorić et al. 2015). Reduced total sleep time has been associated with higher light physical activity (LPA; (Mcneil et al. 2015)) and MVPA (Pesonen et al. 2011; Sorić et al. 2015) the following day. 
Conversely, other research has observed no association between sleep and MVPA (Ekstedt et al. 2013; Krietsch et al. 2016) or energy expenditure (Sorić et al. 2015) the following day.

The lack of consistent findings may be attributable to measurement issues, including differences in device type, device location (wrist, upper arm, waist, and ankle) and measurement periods (1-7 days). While appropriate measurement periods are device dependent, which can also make comparisons between studies difficult, prior research suggests that 3-4 days and 6-7 nights are required for valid accelerometry measured physical activity and sleep outcomes, respectively (Acebo et al. 1999; Ridgers et al. 2016). In addition, there is a lack of consistency concerning the physical activity and sleep outcomes assessed, with some studies focusing solely on MVPA (Krietsch et al. 2016) while others examine all physical activity intensities (Mcneil et al. 2015; Stone et al. 2013). Lastly, it is possible that participant (e.g., age, sex, obesity status) and environmental (e.g., the built environment) factors may influence physical activity and sleep variables. Where possible, these should be controlled for in statistical analyses.

Given the dose response between physical activity and health (Janssen and LeBlanc 2010; Ruiz et al. 2006), it is not surprising that studies investigating the bidirectional relationships between physical activity and sleep have focussed on MVPA. However, few studies have examined the associations between LPA and sleep outcomes (Mcneil et al. 2015) despite LPA accounting for more daily physical activity than MVPA (Owen et al. 2014). Similarly, little research has focused on activity energy expenditure (AEE), which results from all activities of daily living (volitional and structured; Levine 2003). Recent research has shown the health benefits of engaging in LPA and suggest that LPA may provide an initial avenue for sedentary individuals to increase activity on course to meeting physical activity 
recommendations (Carson et al. 2013). Consequently, further research is needed to examine the relationship between LPA and sleep outcomes, as LPA may also be an appropriate and feasible target for interventions in youth. This study aims to contribute to the literature concerning the association between LPA and sleep outcomes. The overall objective of this study was to examine the temporal and bidirectional associations between objectivelymeasured physical activity (of different intensities), AEE and sleep in primary school-aged children.

\section{Methods}

Sample

Primary schools located within a $30 \mathrm{~km}$ radius of Deakin University were identified and randomly invited to participate in the Fitness, Activity and Skills Testing (FAST) Study. In brief, FAST aimed to examine how children's fitness and fundamental movement skills (FMS) influence their day-to-day patterns of physical activity and sedentary time (Ridgers et al. 2016). Six schools located in high socioeconomic status areas (based on the SocioEconomic Index for Areas; Australian Bureau of Statistics 2011) provided informed written Principal consent to participate in the study. Ethical approvals were provided by the Deakin University Human Ethics Advisory Group (Health) and the Catholic Education Office Archdiocese of Melbourne.

All children $(\mathrm{n}=458)$ in Years 4 and 5 (aged 8-11 years) were invited to participate. Onehundred and thirty-eight children ( 68 boys, 70 girls; response rate $=30 \%$ ) returned informed written parental consent to participate in the primary outcome assessments (accelerometry, surveys, fitness, and FMS). A sub-sample of 102 children (51 boys, 51 girls) also provided 
informed written parental consent to wear a SenseWear Armband (BodyMedia Inc, Pittsburgh, USA). In Australia it is an ethical requirement that informed consent is provided, therefore no information was obtained concerning non-responders within schools. Data were collected July to November 2014 (mid-winter to late spring).

\section{Measures}

Anthropometry: At each school, participant's height (to the nearest $0.1 \mathrm{~cm}$ ) and weight (to the nearest $0.1 \mathrm{~kg}$ ) were measured by trained research staff using a portable stadiometer (SECA model 2.17; SECA, Germany) and calibrated digital scales (Tanita BC-351; Tanita, Japan), respectively. Children wore light clothing and no shoes. Waist circumference (to the nearest $\mathrm{cm}$ ) was measured using a flexible steel tape at the narrowest point between the bottom rib and the iliac crest, in the mid-axillary plane. Two measures were taken and in the event of a discrepancy of $0.1 \mathrm{~cm}, 0.1 \mathrm{~kg}$ or $1 \mathrm{~cm}$ for stature, body mass and waist circumference, respectively, a third measure was taken and the average of the two closest measurement recorded. BMI was calculated and converted to BMI z-scores based on the WHO international growth reference charts (de Onis et al. 2007).

Physical activity and sleep: Free-living physical activity and sleep were objectively-assessed using the SenseWear Pro Armband (BodyMedia Inc., Pittsburgh, USA). The SenseWear is a multi-sensor monitor (triaxial accelerometer, heat flux, galvanic skin response, skin temperature, and near-body ambient temperature sensors) and is worn on the left upper arm over the triceps muscle. The SenseWear is currently the only device that has been validated against gold standard measures of both physical activity (doubly-labelled water and indirect calorimetry in free-living and laboratory conditions; Calabro et al. 2013; Lee et al. 2016) and sleep (polysomnography for total sleep time; Roane et al. 2015; Soric et al. 2013). Each 
monitor was configured with the child's date of birth, sex, handedness, stature and body mass using proprietary software (SenseWear Professional v7, BodyMedia Inc). Physiological data were sampled at a frequency of $32 \mathrm{~Hz}$. Children were instructed to wear the SenseWear Armband for $24 \mathrm{hr} /$ day for eight consecutive days and were provided information about the correct wear and care of the monitor according to manufacturer guidelines. In this sample, monitors were removed for an average of 47 minutes per day for water-based activities (e.g. showering, bathing) and contact sport (e.g. football).

On return, SenseWear data were downloaded and processed in 1 minute epochs using algorithms within the proprietary software. The epoch length is determined by the manufacturer and therefore cannot be changed. Non-wear criteria were not required as the SenseWear Armband can directly identify periods of non-wear through the skin sensors (Johannsen et al. 2010). For the purpose of this study, the following physical activity variables were calculated for each valid day: time spent in light- (LPA; 1.5-3.99 METs), moderate- (MPA; 4-5.99 METs) and vigorous-intensity (VPA; $\geq 6$ METs), and AEE (kcal/day). A threshold of 4 METs was chosen to represent MPA as brisk walking has been associated with an energy cost of 4 METs in calibration studies with children (Trost et al. 2011). MPA and VPA were also summed to obtain time spent in MVPA. Physical activity data were included if children wore the monitor for a minimum of $12 \mathrm{~h}$ on at least 5 days. This provides reliable estimates of all physical activity variables at a reliability of 0.7 (Ridgers et al. 2016). The sleep variables obtained were total sleep time (min/night), time in bed (min/night), and sleep efficiency ((total sleep time/time in bed)*100). A night was scored as valid when overnight data were recorded (Ridgers et al. 2016), with data included if children wore the monitor for 7 nights. These criteria for sleep also provide reliability 
estimates of 0.7 (Ridgers et al. 2016). Children who provided complete valid data for both physical activity and sleep outcomes were included in the analyses.

\section{Statistical Analyses}

Statistical analyses were conducted using Stata 12.0 (Stata Corporation, Texas, USA). Initially, descriptive statistics (mean $\pm \mathrm{SD}$ ) were calculated for all measured variables. Independent t-tests were conducted to examine differences between children with complete and incomplete SenseWear data, and those provided with a SenseWear compared to those that were not. The main analysis consisted of multilevel models that were conducted using the generalised linear latent and mixed models (GLLAMM) procedure. Multilevel models are the most appropriate technique for analysing hierarchical data that are not independent of each other (Twisk 2006). The analyses examined the temporal associations between physical activity and sleep using a three-level model, namely day (Level 1), child (Level 2) and school (Level 3). In all models, the random structure considered random intercepts at the school and child level and random slopes for the outcome variable identified. All analyses were adjusted for age, sex, waist circumference, day, and daily wear time. The first set of analyses examined whether physical activity on any given day was temporally associated with sleep outcomes the following night (307 data points). The second set of analyses examined whether sleep during any given night was temporally associated with physical activity the following day (384 data points). Separate analyses were conducted for each physical activity and sleep outcome for the whole sample. Analyses were conducted on MPA and VPA separately but no significant findings were observed (data not shown), thus combined MVPA is reported. The final parameter estimates are reported in-text as $\beta$ coefficient, confidence interval and $p$ value. Statistical significance was set at $p \leq 0.05$. 


\section{Results}

The subsample of children provided with a SenseWear were significantly taller $(3.4 \mathrm{~cm} ; p<$ $0.05)$, and had a higher body mass $(4.4 \mathrm{~kg} ; p<0.01)$ and BMI z-score $(0.56 ; p<0.01)$ compared to those that did not receive the monitor. Sixty-five children ( 31 boys, 34 girls) had complete physical activity and sleep data and were included in the main analyses. Missing data were attributable to the requirement for 7 nights of sleep data, which provides reliable estimates for both outcomes. There were no significant differences in demographic, physical activity, AEE, and sleep data between children who were included and excluded from the analyses. Demographic information, physical activity levels and sleep outcomes of the children are presented in Table 1 . The majority of the sample (88\%) met the physical activity guidelines at least 60 minutes of MVPA per day (average day method). Three children (4.6\%) met the average total sleep time recommendation of 9-11 $\mathrm{h}$ per night (National Sleep Foundation 2011).

Associations between daily physical activity and nocturnal sleep

When adjusting for participant-level covariates, day, and daily wear time, no significant associations were observed between MVPA and nocturnal sleep outcomes (time in bed, total sleep time, sleep efficiency) (Table 2). Similar findings were observed for LPA and AEE.

Associations between nocturnal sleep and physical activity the following day

When adjusting for participant-level covariates, day, and daily wear time, no associations were observed for nocturnal sleep on next day physical activity and AEE across the whole sample (Table 2) for all assessed outcomes.

\section{Discussion}


This study is one of the first to examine the temporal and bidirectional relationships between LPA, MVPA and AEE and sleep outcomes in children. Our results indicate that the total daily volume of LPA and MVPA undertaken on one day was not associated with sleep outcomes. Additionally, no association was observed for AEE on one day and nocturnal sleep outcomes. Similarly, nocturnal time in bed, total sleep time and sleep efficiency were not associated with the total volume of LPA, MVPA, or AEE the following day. This is one of the few studies that have objectively measured physical activity and sleep outcomes using the same device.

The present study observed no associations between MVPA and sleep outcomes, consistent with other studies (Ekstedt et al. 2013; Sorić et al. 2015). Additionally, we observed no association between nocturnal sleep outcomes and MVPA the following day, also consistent with other research (Ekstedt et al. 2013; Krietsch et al. 2016). The non-significant associations between AEE and sleep is also consistent with previous research (Sorić et al. 2015). In contrast to a recent study (McNeil et al. 2015), no significant temporal or bidirectional associations were observed for LPA and sleep outcomes. McNeil and colleagues (2015) noted negative associations between LPA with total sleep time as well as greater LPA in participants with the shortest sleep durations. The difference in these results may be caused by discrepancies between average physical activity and sleep duration levels between samples. Participants in this study were nearly twice as active as those studied by McNeil and colleagues (2015). Further, the average total sleep time in the current study was $1.1 \mathrm{~h}$ less than obtained by those children studied by McNeil and colleagues (2015). More studies are needed to determine the directionality of the relationship between LPA and sleep duration. 
In this study, children were highly active (average of 2hr/day of MVPA) and nearly all participants $(88 \%)$ met the physical activity recommendations. However, only three participants $(5 \%)$ in the sample met the national sleep recommendations of 9-11 h of sleep per night. This finding is of concern, as meta-analyses have found that children who sleep less than the recommended daily amount are at a $58 \%$ increased risk of being overweight or obese (Chen et al. 2008). Previous research has shown that screen time affects sleep onset (Foley et al. 2013), which may in turn be displaced by opportunities provided to engage in structured sports (Hohepa et al. 2009). This may be one explanation for the high levels of activity yet reduced total sleep time in this sample. Sleep efficiency (the percentage of total time in bed actually spent asleep) gives a general estimation of sleep quality (Shrivastava et al. 2014). In the current study, child sleep efficiency was low (81.9\%). This may indicate that children had a long sleep latency and/or long sleep offset to lights on time. More accurate measures of sleep quality are required to further explore the reason for the low sleep efficiency observed.

There is some evidence to suggest that physical activity may improve sleep quality over and above total sleep time (Dworak et al. 2008). To determine sleep quality, the gold standard method, polysomnography, measures brain activity (electroencephalogram) to identify periods of sleep and wake, as well as the individual sleep stages (Kushida et al. 2005). However, to the authors' knowledge no research to date has assessed the impact of daily physical activity on sleep quality. While polysomnography is appropriate for evaluating sleep in laboratory studies, traditional techniques are difficult to employ in free-living environments, as the measurement setup is relatively intrusive, arduous, and usually requires an on-site sleep technician (Ancoli-Israel et al. 2003). Other research has suggested that alternative sleep-related domains such as regularity of a child's sleep schedule (Stone et al. 
2013) and sleep timing (Ekstedt et al. 2013; Krietsch et al. 2016) may be more strongly related to children's physical activity the following day than total sleep time. For example, Krietsch and colleagues (2016) noted that later sleep onset times but not total sleep time was the strongest predictor of group-level decreases in physical activity in overweight and obese children. Collectively, these findings suggest that future research should focus on measures of sleep quality, the regularity of sleep patterns and sleep timing (as opposed to total sleep time) across the weight continuum in children and how these impact next day physical activity.

The current study is one of few that have used valid objective measurement of physical activity and sleep in a free-living environment for multiple days using the same device in primary school-aged children. The diverse range of measurement devices employed when evaluating child physical activity and sleep makes comparisons between studies difficult. Thus, standardized measurement practices are warranted for assessment, cleaning and reporting of such data. Future research could benefit from compositional analyses, which recently have been shown to be superior to traditional linear regression techniques as $24-\mathrm{h}$ behavioural data (sleep, sedentary time and physical activity) are intrinsically co-dependent, finite and subject to collinearity (Carson et al. 2016; Chastin et al. 2015). The present findings are limited to Australian children aged 8-11 years old who attended highsocioeconomic schools in the Melbourne region. This may limit the generalizability of these results to low/mid-socioeconomic and younger primary school children. In the current study, child and/or parental reported sleep onset and offset times were not collected. Such information could have been beneficial to corroborate or guide identification of sleep periods (as opposed to periods of quiescent wakefulness), as well as identify artefacts in the SenseWear output (Meltzer et al. 2012). Secondly, this study did not examine these associations for weekdays and weekend days separately due to the small sample sized 
retained. Future studies should determine whether such associations are consistent for weekdays and weekend days given the variability in waking and sleeping times across these periods of the week. Finally, temporal associations using cross-sectional data does not equate to causality.

The current study objectively assessed the temporal and bidirectional relationships between physical activity, AEE and sleep. No significant associations were observed between physical activity, AEE and sleep in either direction. Future research is needed to understand whether other sleep dimensions (e.g., quality, regularity, timing) may be impacted by or may influence physical activity.

\section{Conflict of interest}

The authors report no conflicts of interest associated with this manuscript.

\section{Acknowledgements}

The authors gratefully acknowledge the contribution of all project staff, especially Winsfred Ngan, Sam Lai, Natalie Lander, Christine Minto and Christine Granger.

\section{Funding}

This study was funded by the Deakin University Central Research Grants Scheme [RM27684]. GV is supported by an Early Career Fellowship from Central Queensland University. LMB is supported by an Alfred Deakin Postdoctoral Research Fellowship; DRL is supported by an Australian Research Council Future Fellowship [FT140100399]; AT is supported by a Future Leader Fellowship from the National Heart Foundation of Australia [Award ID 100046]; JS is supported by a National Health and Medical Research Council 
Principal Research Fellowship [APP1026216]. NDR was supported by an Australian Research Council Discovery Early Career Researcher Award [DE120101173]. 


\section{References}

Acebo, C., Sadeh, A., Seifer, R., Tzischinsky, O., Wolfson, A., Hafer, A., et al. 1999.

Estimating sleep patterns with activity monitoring in children and adolescents: how many nights are necessary for reliable measures? Sleep 22(1): 95-103. PMID: 9989370.

Ancoli-Israel, S., Cole, R., Alessi, C., Chambers, M., Moorcroft, W., and Pollak, C. 2003. The role of actigraphy in the study of sleep and circadian rhythms. Sleep 26(3): 342-392. PMID: 12749557.

Andersen, L.B., Harro, M., Sardinha, L.B., Froberg, K., Ekelund, U., Brage, S., et al. 2006. Physical activity and clustered cardiovascular risk in children: a cross-sectional study (The European Youth Heart Study). The Lancet 368(9532): 299-304. doi: 10.1016/S01406736(06)69075-2. PMID: 16860699.

Australian Bureau of Statistics. 2011. Census of Population and Housing: Socio-Economic Indexes for Areas (SEIFA), Australia, 2011 Available from http://www.abs.gov.au/ausstats/abs@.nsf/mf/2033.0.55.001 [accessed 1st May 2012].

Calabro, M.A., Stewart, J.M., and Welk, G.J. 2013. Validation of pattern-recognition monitors in children using doubly labeled water. Med. Sci. Sports Exerc. 45(7): 1313-1322. doi: 10.1249/MSS.0b013e31828579c3. PMID: 23299766.

Carson, V., Ridgers, N.D., Howard, B.J., Winkler, E.A., Healy, G.N., Owen, N., et al. 2013. Light-intensity physical activity and cardiometabolic biomarkers in US adolescents. PLoS One 8(8): e71417. doi: 10.1371/journal.pone.0071417. PMID: 23951157.

Chen, X., Beydoun, M.A., and Wang, Y. 2008. Is sleep duration associated with childhood obesity? A systematic review and meta-analysis. Obesity 16(2): 265-274. doi:

10.1038/oby.2007.63. PMID: 18239632. 
de Onis, M., Onyango, A.W., Borghi, E., Siyam, A., Nishida, C., and Siekmann, J. 2007. Development of a WHO growth reference curve for school-aged children and adolescents. Bull. World Health Organ. 85(9): 660-667. PMID: 18026621.

Dollman, J., Norton, K. and Norton, L. 2005. Evidence for secular trends in children's physical activity behaviour. Br. J. Sports Med. 39(12): 892-897. doi:

10.1136/bjsm.2004.016675. PMID: 16306494.

Dworak, M., Wiater, A., Alfer, D., Stephan, E., Hollmann, W., and Strüder, H.K. 2008. Increased slow wave sleep and reduced stage 2 sleep in children depending on exercise intensity. Sleep Med. 9(3): 266-272. doi: 10.1016/j.sleep.2007.04.017. PMID: 17644426. Ekstedt, M., Nyberg, G., Ingre, M., Ekblom, O., and Marcus, C. 2013. Sleep, physical activity and BMI in six to ten-year-old children measured by accelerometry: A crosssectional study. Int. J. Behav. Nutr. Phys. Act. 10: 82. doi: 10.1186/1479-5868-10-82. PMID: 23800204.

Foley, L.S., Maddison, R., Jiang, Y., Marsh, S., Olds, T., and Ridley, K. 2013. Presleep activities and time of sleep onset in children. Pediatrics 131(2): 276-282. doi: 10.1542/peds.2012-1651. PMID: 23319532.

Hart, C.N., Hawley, N., Davey, A., Carskadon, M., Raynor, H., Jelalian, E., et al. 2016. Effect of experimental change in children's sleep duration on television viewing and physical activity. Pediatr. Obes. doi: 10.1111/ijpo.12166. PMID: 27417142.

Hillman, D and Lack, L. 2013. Public health implications of sleep loss: the community burden. Med. J. Aust. 199(8): S7-S10. PMID: 24138358.

Hohepa, M., Scragg, R., Schofield, G., Kolt, G.S., and Schaaf, D. 2009. Associations between after-school physical activity, television use, and parental strategies in a sample of New Zealand adolescents. J. Phys. Act. Health 6(3): 299-305. PMID: 19564657. 
Janssen, I., and LeBlanc, A.G. 2010. Systematic review of the health benefits of physical activity and fitness in school-aged children and youth. Int. J. Behav. Nutr. Phys. Act. 7(40): 1-16. doi: 10.1186/1479-5868-7-40. PMID: 20459784.

Johannsen, D.L., Calabro, M.A., Stewart, J., Franke, W., Rood, J.C., and Welk, G.J. 2010. Accuracy of armband monitors for measuring daily energy expenditure in healthy adults. Med. Sci. Sports Exerc. 42(11): 2134-2140. doi: 10.1249/MSS.0b013e3181e0b3ff. PMID: 20386334.

Krietsch, K.N., Armstrong, B., McCrae, C.S., and Janicke, D.M. 2016. Temporal associations between sleep and physical activity among overweight/obese youth. J. Pediatr. Psychol. 41(6): 680-691. doi: 10.1093/jpepsy/jsv167. PMID: 26801238.

Kushida, C.A., Littner, M.R., Morgenthaler, T., Alessi, C.A., Bailey, D., Coleman Jr, J., et al. 2005. Practice parameters for the indications for polysomnography and related procedures: An update for 2005. Sleep 28(4): 499-521. PMID: 16171294.

Lee, J.M., Kim, Y., Bai, Y., Gaesser, G.A., and Welk, G.J. 2016. Validation of the SenseWear mini armband in children during semi-structure activity settings. J. Sci. Med. Sport 19(1): 41-45. doi: 10.1016/j.jsams.2014.10.004. PMID: 25459233.

Levine, J.A.. 2003. Non-exercise activity thermogenesis. Proc. Nutr. Soc. 62(3):667-679. PMID: 14692603.

Matricciani, L., Olds, T., and Petkov, J. 2012. In search of lost sleep: secular trends in the sleep time of school-aged children and adolescents. Sleep Med. Rev. 16(3): 203-211. doi: 10.1016/j.smrv.2011.03.005. PMID: 21612957.

Mcneil, J., Tremblay, M.S., Leduc, G., Boyer, C., Bélanger, P., Leblanc, A.G., et al. 2015. Objectively-measured sleep and its association with adiposity and physical activity in a sample of Canadian children. J. Sleep Res. 24(2): 131-139. doi: 10.1111/jsr.12241. PMID: 25266575 . 
Meltzer, L.J., Montgomery-Downs, H.E., Insana, S.P., and Walsh, C.M. 2012. Use of actigraphy for assessment in pediatric sleep research. Sleep Med. Rev. 16(5): 463-475. doi: 10.1016/j.smrv.2011.10.002. PMID: 22424706.

National Sleep Foundation. 2011. Children and sleep —information on sleep health and safety. Available from http://www.sleepfoundation .org/article/sleep-topics/children-andsleep. [accessed 1st May 2014].

Owen, N., Salmon, J., Koohsari, M.J., Turrell, G., and Giles-Corti, B. 2014. Sedentary behaviour and health: mapping environmental and social contexts to underpin chronic disease prevention. Br. J. Sports Med. 48(3): 174-177. doi: 10.1136/bjsports-2013-093107. PMID: 24415410.

Pesonen, A.-K., Sjöstén, N.M., Matthews, K.A., Heinonen, K., Martikainen, S., Kajantie, E., et al. 2011. Temporal associations between daytime physical activity and sleep in children. PLoS One 6(8): e22958. doi: 10.1371/journal.pone.0022958. PMID: 21886770.

Ridgers, N.D., Hnatiuk, J.A., Vincent, G.E., Timperio, A., Barnett, L.M., and Salmon, J. 2016. How many days of monitoring are needed to reliably assess SenseWear Armband outcomes in primary school-aged children? J. Sci. Med. Sport doi: 10.1016/j.jsams.2016.02.009. PMID: 26971298.

Roane, B.M., Van Reen, E., Hart, C.N., Wing, R., and Carskadon, M.A. 2015. Estimating sleep from multisensory armband measurements: validity and reliability in teens. J. Sleep Res. 24(6): 714-721. doi: 10.1111/jsr.12317. PMID: 26126746.

Ruiz, J.R., Rizzo, N.S., Hurtig-Wennlöf, A., Ortega, F.B., Wärnberg, J., and Sjöström, M. 2006. Relations of total physical activity and intensity to fitness and fatness in children: the European Youth Heart Study. Am. J. Clin. Nutr. 84(2): 299-303. PMID: 16895875. 
Sekine, M., Yamagami, T., Handa, K., Saito, T., Nanri, S., Kawaminami, K., et al. 2002. A dose-response relationship between short sleeping hours and childhood obesity: results of the Toyama Birth Cohort Study. Child Care Health Dev. 28(2): 163-170. PMID: 11952652. Shrivastava, D., Jung, S., Saadat, M., Siohi, R., Crewson, K. 2014. How to interpret the results of a sleep study. J. Community Hosp. Intern. Med. Perspect. 2014, 4(5): 24983. doi: 10.3402/jchimp.v4.24983. PMID: 25432643.

Sorić, M., Starc, G., Borer, K.T., Jurak, G., Kovač, M., Strel, J., et al. 2015. Associations of objectively assessed sleep and physical activity in 11-year old children. Ann. Hum. Biol. 42(1): 31-37. doi: 10.3109/03014460.2014.928367. PMID: 25007958.

Sorić, M., Turkalj, M., Kucic, D., Marusic, I., Plavec, D., and Misigoj-Durakovid, M. 2013. Validation of a multi-sensor activity monitor for assessing sleep in children and adolescents. Sleep Med. 14: 201-205. doi: 10.1016/j.sleep.2012.11.003. PMID: 23238268.

Spruyt, K., Molfese, D.L., and Gozal, D. 2011. Sleep duration, sleep regularity, body weight, and metabolic homeostasis in school-aged children. Pediatrics 127(2): e345-e352. doi: 10.1542/peds.2010-0497. PMID: 21262888.

Stone, M.R., Stevens, D., and Faulkner, G.E. 2013. Maintaining recommended sleep throughout the week is associated with increased physical activity in children. Prev. Med. 56(2): 112-117. doi: 10.1016/j.ypmed.2012.11.015. PMID: 23201000.

Taheri, S. 2006. The link between short sleep duration and obesity: we should recommend more sleep to prevent obesity. Arch. Dis. Child. 91(11): 881-884. doi:

10.1136/adc.2005.093013. PMID: 17056861.

Touchette, E., Petit, D., Tremblay, R.E., Boivin, M., Falissard, B., Genolini, C., et al. 2008. Associations between sleep duration patterns and overweight/obesity at age 6. Sleep 31(11): 1507-1514. PMID: 19014070. 
Trost, S.G., Loprinzi, P.D., Moore, R., and Pfeiffer, K.A. 2011. Comparison of accelerometer cut points for predicting activity intensity in youth. Med. Sci. Sports Exerc. 43(7): 13601368. doi: 10.1249/MSS.0b013e318206476e. PMID: 21131873.

Twisk, J.W.R. 2006. Applied Multilevel Analysis: A Practical Guide. Cambridge University Press Cambridge, Cambridge, UK.

World Health Organization. 2010. Global recommendations on physical activity for health. Geneva, World Health Organization. 
Table 1: Descriptive, physical activity, and sleep characteristics of included children

\begin{tabular}{lc}
\hline & Whole sample \\
& $(\mathbf{n}=\mathbf{6 5})$ \\
\hline Age (y) & $10.4 \pm 0.6$ \\
Stature (cm) & $143.0 \pm 7.6$ \\
Body mass (kg) & $36.9 \pm 7.3$ \\
z-BMI & $0.54(0.86)$ \\
Waist circumference (cm) & $65.6 \pm 7.8$ \\
Total LPA (min/day) & $378.5 \pm 70.7$ \\
Total MPA (min/day) & $92.3 \pm 37.5$ \\
Total VPA (min/day) & $29.6 \pm 23.6$ \\
Total MVPA (min/day) & $121.9 \pm 55.3$ \\
Activity energy expenditure (kcal/day) & $1003.4 \pm 229.1$ \\
Total sleep time (min/night) & $475.5 \pm 35.4$ \\
Time in bed (min/night) & $580.5 \pm 33.6$ \\
Weep efficiency (\%) & $1392.7(39.1)$ \\
\hline Data are prime (min/day) & \\
\hline
\end{tabular}

Data are presented as mean $\pm \mathrm{SD}$. 
Table 2. Within-participant associations between (a) daily physical activity and nocturnal sleep and (b) nocturnal sleep and physical activity the following day $(n=65)$

b $(95 \%$ CI $) \quad$ v value

(a) Associations between daily physical activity and sleep
MVPA (min) $\rightarrow$ TIB (min)
$-0.06(-0.13$ to 0.03$)$
0.26
LPA (min) $\rightarrow$ TIB (min)
$-0.04(-0.11$ to 0.03$)$
0.23
AEE (kcal) $\rightarrow$ TIB (min)
$-0.02(-0.04$ to 0.01$)$
0.07
MVPA (min) $\rightarrow$ TST (min)
$-0.05(-0.14$ to 0.03$)$
0.20
LPA $(\min ) \rightarrow$ TST (min)
$0.01(-0.06$ to 0.07$)$
0.87
$\operatorname{AEE}(\mathrm{kcal}) \rightarrow \mathrm{TST}(\mathrm{min})$
$-0.01(-0.04$ to 0.01$)$
0.18
MVPA (min) $\rightarrow$ SE (\%)
$-0.01(-0.01$ to 0.1$)$
0.98
$\mathrm{LPA}(\min ) \rightarrow \mathrm{SE}(\%)$
$0.01(-0.01$ to 0.01$)$
0.17
$\mathrm{AEE}(\mathrm{kcal}) \rightarrow \mathrm{SE}(\%)$
$0.01(-0.01$ to 0.01$)$
0.73

(b) Associations between nocturnal sleep and

physical activity the following day

\begin{tabular}{|c|c|c|c|c|}
\hline TIB (min) & $\rightarrow$ & MVPA (min) & $-0.08(-0.23$ to 0.10$)$ & 0.41 \\
\hline TIB (min) & $\rightarrow$ & LPA (min) & $0.05(-0.14$ to 0.19$)$ & 0.77 \\
\hline TIB (min) & $\rightarrow$ & AEE (kcal) & $-0.22(-0.93$ to 0.49$)$ & 0.54 \\
\hline TST (min) & $\rightarrow$ & MVPA (min) & $-0.09(-0.22$ to 0.04$)$ & 0.19 \\
\hline TST (min) & $\rightarrow$ & LPA (min) & $0.04(-0.23$ to 0.12$)$ & 0.53 \\
\hline TST (min) & $\rightarrow$ & AEE (kcal) & $-0.34(-0.99$ to 0.32$)$ & 0.32 \\
\hline SE $(\%)$ & $\rightarrow$ & MVPA (min) & $0.11(-1.02$ to 1.26$)$ & 0.83 \\
\hline SE $(\%)$ & $\rightarrow$ & LPA (min) & $-0.69(-2.08$ to 0.69$)$ & 0.13 \\
\hline SE (\%) & $\rightarrow$ & AEE (kcal) & $-1.04(-5.9$ to 3.8$)$ & 0.68 \\
\hline
\end{tabular}


Adjusted for age, sex, waist circumference, day, and daily wear time.

Abbreviations: TIB, time in bed; TST, total sleep time; SE, sleep efficiency; LPA, lightintensity physical activity, MVPA, moderate-vigorous intensity physical activity. 